\title{
Quantitative measurement of voltage dependence of spin-transfer torque in MgO-based magnetic tunnel junctions
}

\author{
HITOSHI KUBOTA ${ }^{1 *}$, AKIO FUKUSHIMA ${ }^{1}$, KAY YAKUSHIJI ${ }^{1}$, TARO NAGAHAMA ${ }^{1}$, SHINJI YUASA ${ }^{1}$, \\ KOJI ANDO1 ${ }^{1}$, HIROKI MAEHARA², YOSHINORI NAGAMINE², KOJI TSUNEKAWA², \\ DAVID D. DJAYAPRAWIRA², NAOKI WATANABE² AND YOSHISHIGE SUZUKI ${ }^{1,3}$
}

\author{
${ }^{1}$ National Institute of Advanced Industrial Science and Technology (AIST), Nanoelectronics Research Institute (NeRI), Tsukuba, Ibaraki 305-8568, Japan \\ ${ }^{2}$ Electron Device Division, Canon ANELVA Corporation, Fuchu, Tokyo 183-8508, Japan \\ ${ }^{3}$ Graduate School of Engineering Science, Osaka University, Toyonaka, Osaka 560-8531, Japan \\ *e-mail: hit-kubota@aist.go.jp
}

When an electric current passes from one ferromagnetic layer via a non-magnetic layer into another ferromagnetic layer, the spin polarization and subsequent rotation of this current can induce a transfer of angular momentum that exerts a torque on the second ferromagnetic layer ${ }^{1-4}$. This provides a potentially useful method to reverse $e^{3,5-7}$ and oscillate ${ }^{8}$ the magnetic momenta in nanoscale magnetic structures. Owing to the large current densities required to observe spin-torqueinduced magnetization switching and microwave emission $\left(\sim 10^{7} \mathrm{~A} \mathrm{~cm}^{-2}\right)$, accurately measuring the strength, or even the direction, of the associated spin torque has proved difficult. Yet, such measurements are crucial to refining our understanding of the mechanisms responsible and the theories that describe them ${ }^{9,10}$. To address this, we present quantitative experimental measurements of the spin torque in $\mathrm{MgO}$-based magnetic tunnel junctions ${ }^{11-14}$ for a wide range of bias currents covering the switching currents. The results verify the occurrence of two different spin-torque regimes with different bias dependences that agree well with theoretical predictions ${ }^{10}$.

Magnetic tunnel junctions (MTJs) consisting of a $\mathrm{MgO}$ insulating layer sandwiched between two ferromagnetic layers $\left(\mathbf{S}_{1}\right.$ and $\mathbf{S}_{2}$ in Fig. 1a) were used to provide very large magnetoresistance ${ }^{11,14}$. Such MTJs are now useful as data storage cells in magnetic random-access memories (M-RAMs) and as magnetic-field sensors in magnetic hard disk drives ${ }^{11-13}$. The MTJs with a layer structure of $\mathrm{Ir}-\mathrm{Mn} / \mathrm{Co}-\mathrm{Fe} / \mathrm{Ru} / \mathrm{Co}_{60} \mathrm{Fe}_{20} \mathrm{~B}_{20} / \mathrm{MgO} / \mathrm{Co}_{60} \mathrm{Fe}_{20} \mathrm{~B}_{20}$ were prepared on a $\mathrm{MgO}$ substrate using an ultrahigh-vacuum sputtering system (C-7100; Canon ANELVA). The 3-nm-thick bottom Co-Fe-B layer $\left(\mathbf{S}_{1}\right)$ acts as a spin polarizer. The top Co-Fe-B layer $\left(\mathbf{S}_{2}\right)$, a 2 -nm-thick free layer, is excited by the spin torque. The $\mathrm{MgO}$ tunnel barrier is about $1 \mathrm{~nm}$ thick. The MTJs are rectangular with dimensions of approximately $70 \mathrm{~nm} \times 250 \mathrm{~nm}$ (see the Methods section for preparation details).

Resistance-magnetic-field $(R-H)$ curves measured at a small bias voltage $(0.1-0.3 \mathrm{mV})$ and different in-plane field directions, that is, $\theta_{\mathrm{H}}=0$ and $45^{\circ}$, are shown in Fig. 1b. $\theta_{\mathrm{H}}$ is the angle between the applied field direction and the easy axis of the magnetic cell along the long axis of the rectangular cell (see Fig. 1a). The magnetoresistance ratio is defined as $\mathrm{MR}=\left(R_{\mathrm{AP}}-R_{\mathrm{P}}\right) / R_{\mathrm{P}}$, where $R_{\mathrm{P}}$ and $R_{\mathrm{AP}}$ respectively represent resistance in the parallel and antiparallel magnetization alignments of $\boldsymbol{S}_{1}$ and $\boldsymbol{S}_{2}$. A positive bias current denotes electron flow from $S_{2}$ to $S_{1}$. The magnetoresistance ratio and $R_{\mathrm{P}}$ at a small bias voltage are, respectively, $154 \%$ and about $120 \Omega\left(R_{\mathrm{P}} \times\right.$ (Junction area $\left.)=2 \Omega \mu \mathrm{m}^{2}\right)$. Figure 1c shows the bias voltage, $V_{\mathrm{b}}$, dependence of the tunnelling resistance, as measured in four different fields (A-D), which are indicated by arrows in Fig. 1b. For antiparallel alignment (curves A and B), the resistance decreases with increasing $V_{\mathrm{b}}$ because new tunnelling channels open at higher bias voltages ${ }^{15}$. In contrast, for parallel alignments ( $\mathrm{C}$ and $\mathrm{D})$, resistances remain almost constant, as is commonly observed in $\mathrm{MgO}$-based $\mathrm{MTJs}^{16}$. For curve $\mathrm{B}$, angle $\theta_{12}$ is defined as the angle between $\boldsymbol{S}_{2}$ and $\boldsymbol{S}_{1}$, which is calculable from the resistance value by assuming a cosine dependence of tunnel conductance on $\theta_{12}$ (ref. 17). At the null-field condition, a spin-torque-induced magnetization reversal from antiparallel to parallel (parallel to antiparallel) takes place at a $V_{\mathrm{b}}$ of about $-270 \mathrm{mV}(+380 \mathrm{mV})$ (see Supplementary Information, Fig. S1).

We used the spin-torque diode effect ${ }^{18,19}$ to quantitatively examine the spin torque directly under various biases (see Note added in proof). To measure the effect, a low-amplitude highfrequency current is applied to an MTJ, which exerts an alternative spin torque on $S_{2}$. Owing to the oscillating torque, $S_{2}$ oscillates at the same frequency and, owing to the dependence of the resistance on the angle between $S_{1}$ and $S_{2}$, partially rectifies the applied current. This rectification effect is a kind of homodyne detection: it provides the d.c. output voltage that reflects the spin-torque's size and phase. In our first experiment ${ }^{18}$, we described the existence of two kinds of torque at zero bias: a spin-transfer torque (STT) that rotates spin $\left(\mathbf{S}_{2}\right)$ in the in-plane direction and plays a dominant role in magnetization reversal and a field-like torque (FLT) that rotates $S_{2}$ perpendicularly to the film plane.

Measurements were carried out at room temperature under d.c. bias currents of -1.5 to $+1.5 \mathrm{~mA}$ and a magnetic field $\left(H=-400 \mathrm{Oe}, \theta_{\mathrm{H}}=45^{\circ}\right)$ using the circuit shown in the inset of Fig. 2b (see the Methods section). Observed spin-torque diode 

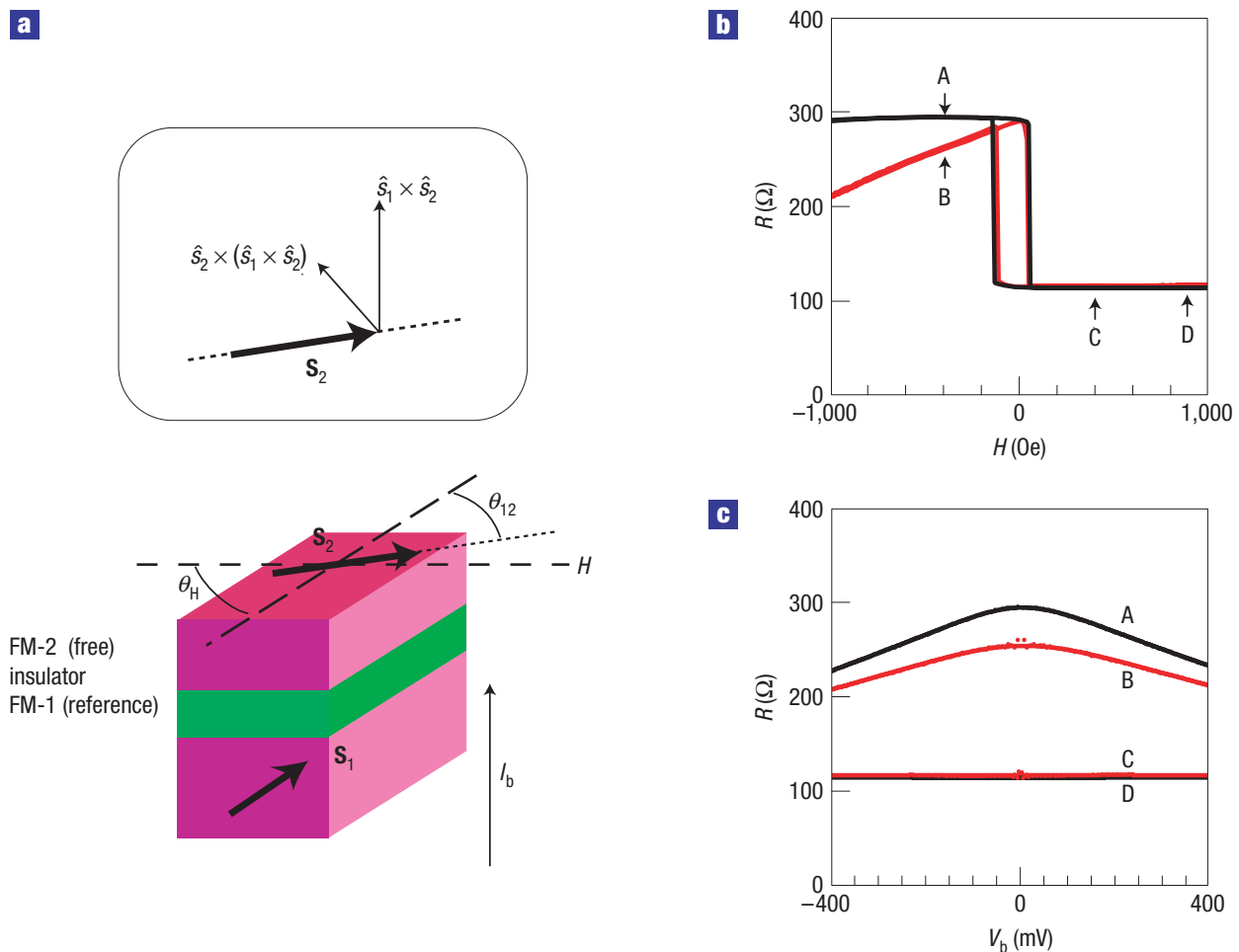

Figure 1 Magnetoresistive properties of the Co-Fe-B/Mg0/Co-Fe-B MTJ. a, Schematic diagram of the MTJ layer structure. FM-1 is magnetically hardened so that $\mathbf{S}_{1}$ does not change its direction even if a strong magnetic field is applied. Electrons passed through FM-1 are spin polarized along the $\mathbf{S}_{1}$ direction. Local spins in the FM-2, $\mathbf{S}_{2}$ align along the long axis of the MTJ cell in a zero magnetic field. In fact, $\mathbf{S}_{2}$ changes its direction freely by applying a magnetic field or spin-polarized currents. An arrow shows the positive bias current in the $\left(l_{b}\right)$ direction. b. External field $(H)$ dependence of the tunnel resistance $(R)\left(R-H\right.$ curve) of the MTJ measured at $\theta_{H}=0$ (black line) and at $45^{\circ}$ (red line). The angle formed by $H$ and the long axis of the MTJ cell is $\theta_{H}$. Here, $\theta_{12}$ is the angle between $\mathbf{S}_{2}$ and $\mathbf{S}_{1}$. Tunnel resistance (excluding the electrical lead resistance) is greatest $\left(R_{A \mathrm{P}}=294 \Omega\right)$ when $\mathbf{S}_{2}$ aligns antiparallel to $\mathbf{S}_{1}$. Tunnel resistance is smallest $\left(R_{\mathrm{p}}=113 \Omega\right)$ when $\mathbf{S}_{2}$ aligns parallel to $\mathbf{S}_{1}$. C, Bias voltage $\left(V_{\mathrm{b}}\right)$ dependence of the tunnel resistance $(R)$. Curves A-D were measured at the external fields indicated by the corresponding arrows in $\mathbf{b}$. Curves $A$ and $B$ respectively correspond to $\theta_{12}=180$ and about $137^{\circ}$.

spectra are shown in Fig. 2a and b, respectively corresponding to negative and positive bias regions. Background signals caused by nonlinear $I-V$ characteristics of the tunnelling conductance were subtracted by assuming a linear frequency dependence around the peaks. In Fig. 2a,b, the peak height varies with the bias. The peak shape is symmetric at zero bias, but it is antisymmetric at large bias magnitudes. The symmetric (antisymmetric) shape of the spectra is a result of the STT (FLT). It should be noted that many other MTJs similar to that used in this experiment showed more complicated spectra, which is thought to be attributable mainly to an uncontrolled magnetization distribution inside the magnetic cell. In our previous paper, the peak was slightly antisymmetric, which can also be explained in a similar way ${ }^{18}$. For samples that show a simple spectrum, the change of the spectrum shape with respect to the bias voltage is quite reproducible and reflects intrinsic features of the spin-torque mechanism.

To evaluate contributions from STT and FLT separately, the spectra were analysed on the basis of a macrospin model in which the distribution of the local magnetization inside the cell is neglected. It is noteworthy that current-induced static fields or non-uniform current flow might violate this assumption under very high d.c. bias currents. The motion of $\boldsymbol{S}_{2}$ is described using the Landau-Lifshitz-Gilbert equation with spin torque ${ }^{1,20}$ :

$$
\frac{\mathrm{d} \hat{s}_{2}}{\mathrm{~d} t}=\gamma \hat{s}_{2} \times \mathbf{H}_{\mathrm{eff}}-\alpha \hat{s}_{2} \times \frac{\mathrm{d} \hat{s}_{2}}{\mathrm{~d} t}+\beta_{\mathrm{ST}} I \hat{s}_{2} \times\left(\hat{s}_{1} \times \hat{s}_{2}\right)+\beta_{\mathrm{FT}} I \hat{s}_{1} \times \hat{s}_{2} .
$$

$\gamma$ is the gyromagnetic ratio and $\hat{s}_{2}$ and $\hat{s}_{1}$ respectively denote the unit vectors parallel to the average spin direction in the free $\left(\mathbf{S}_{2}\right)$ and reference $\left(\mathbf{S}_{1}\right)$ layers. The first term is the field torque; the second term is the damping torque. The third and fourth terms are spin torques: $\beta_{\mathrm{ST}}$ and $\beta_{\mathrm{FT}}$ are coefficients of torque originating, respectively, from STT and FLT. In addition, $\alpha$ is a damping factor and $H_{\text {eff }}$ is an effective field around which $\mathbf{S}_{2}$ precesses. In the experiment, $I$ corresponds to the sum of the d.c. bias current $\left(I_{\mathrm{b}}\right)$ and the high-frequency current $\left(I_{\omega} \sin \omega t\right)$. On the basis of equation (1), the spin-torque diode spectrum can be expressed $\mathrm{as}^{21}$

$$
\begin{aligned}
V_{\text {diode }}(\omega)= & \frac{1}{4} \eta \frac{R_{\mathrm{AP}}-R_{\mathrm{P}}}{R_{\mathrm{P}} R_{\mathrm{AP}}} Z_{0} R_{0} I_{\omega}^{2} \sin ^{2} \theta_{12} \\
& \times f\left[\omega ; \beta_{\mathrm{ST}}, \beta_{\mathrm{FT}}, \omega_{0}, \Delta, I_{\mathrm{b}}, H, H_{\mathrm{d}}, \theta_{12}\right],
\end{aligned}
$$

where $R_{0}$ is the MTJ's resistance at $\theta_{12}$ under $V_{\mathrm{b}}$ and $\eta$ is a factor to correct for high-frequency current attenuation, as determined by another set of experiments. In addition, $Z_{0}(=50 \Omega)$ is the characteristic impedance of cables (see Supplementary Information, Fig. S2). The last factor in equation (2), $f$, describes the shape of the spectrum (see the Methods section). $H_{\mathrm{d}}(=1.38 \mathrm{~T}$ ) and $\omega$ respectively denote the out-of-plane demagnetization field and the angular frequency of the input high-frequency current.

We used equation (2) to fit the experimental results by choosing $\beta_{\mathrm{ST}}, \beta_{\mathrm{FT}}$, resonance angular frequency $\omega_{0}$ and spectrum 

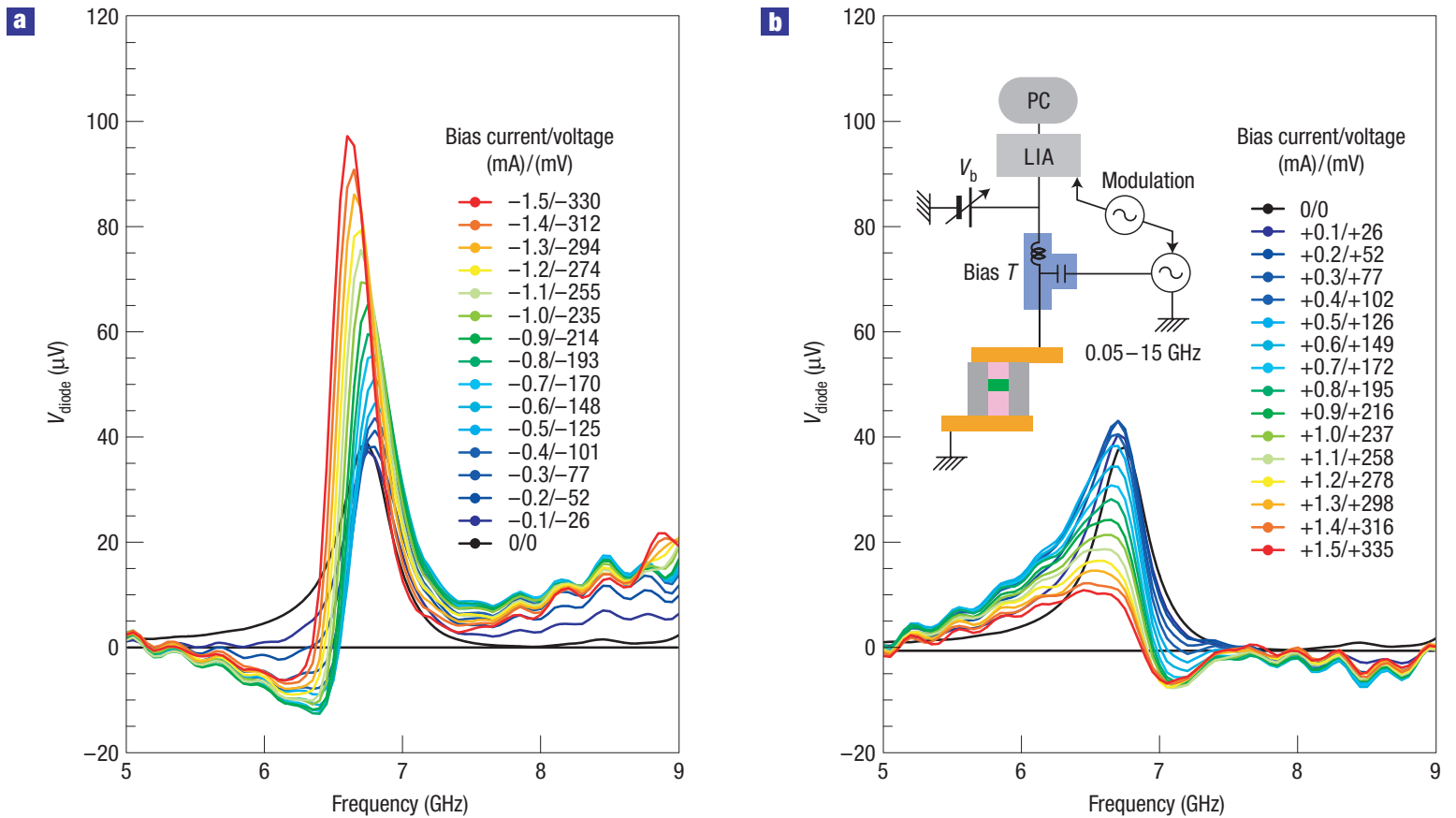

Figure 2 Spin-torque diode spectra measured under various d.c. bias voltages. a,b, Negative bias (a), positive bias (b); in addition, $H=-4000$ e (B in Fig. 1 b) and $\theta_{12}$ of about $137^{\circ}$. The inset in $\mathbf{b}$ shows the measurement circuit for the spin-torque diode effect measurement (LIA: lock-in amplifier). Smooth background voltage was subtracted. Small-amplitude oscillations beside the main peaks are regarded as noise.

linewidth $\Delta$ (see the Methods section) as fitting parameters. Typical fitting results are shown in Fig. 3. Peak shapes are well fitted in every case. Figure 4 shows that both STT and FLT have non-monotonic bias dependence. Near zero bias, the STT shows a linear dependence on bias, as implied from a physical picture in which the spintransfer efficiency is constant. At large negative biases, however, the STT increases rapidly, with higher spin-transfer efficiency for larger negative bias. More surprisingly, for positive bias voltage, the STT shows a minimum around $+250 \mathrm{mV}$ and increases again at biases greater than $+250 \mathrm{mV}$. This strong nonlinear behaviour can be understood by taking a nonlinear bias dependence of each spin-transfer channel in the MTJ, as explained later. In contrast, the magnitude of FLT is smaller than that of STT and shows symmetric dependence on the bias. The linewidth, which reflects the effective damping parameter, changes remarkably in negative bias, consistent with a large change of spin-transfer torque (see Supplementary Information, Fig. S3 for the bias dependence of these parameters).

Theory suggests that the STT acting on $S_{2}$ is equal to the transverse component of the injected spin currents. Consequently ${ }^{9,22}$,

$$
\text { spin torque }=\frac{\hbar}{4 e}\left(G_{+,+}+G_{+,-}-G_{-,-}-G_{-,+}\right) \sin \theta_{12} V_{\mathrm{b}} .
$$

Here, $G_{\sigma_{2}, \sigma_{1}}$ is the conductance of spin subchannels ( + , majority spin; -, minority spin) between $\boldsymbol{S}_{1}$ and $\boldsymbol{S}_{2}$. The total resistance can be expressed as $R_{\mathrm{P}}^{-1}=\left(G_{+,+}+G_{-,-}\right)$and $R_{\mathrm{AP}}^{-1}=\left(G_{+,-}+G_{-,+}\right)$. For an MTJ with symmetrical potential shape with identical electrode materials, as in our case, the contribution from $\left(G_{+,+}-G_{-,-}\right) V_{\mathrm{b}}$ in the equation shown above is inferred to have antisymmetric bias voltage dependence, although that from $\left(G_{+,-}-G_{-,+}\right) V_{\mathrm{b}}$ is symmetrical. Because $R_{\mathrm{AP}}$ has considerable

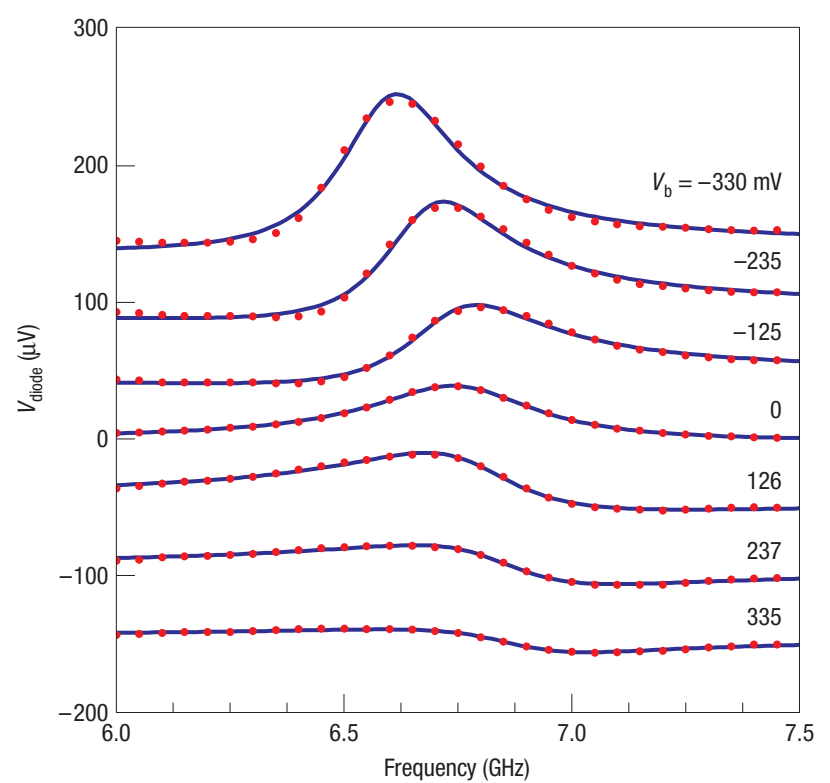

Figure 3 Typical results of theoretical fit based on equation (1). For fitting, we fixed $H_{\mathrm{d}}=1.38 \mathrm{~T}$ and treated $\beta_{\mathrm{ST}}^{\prime}, \beta_{\mathrm{F}}^{\prime}, \omega_{0}$ and $\Delta$ as fitting parameters. The antisymmetry of the peak shape is attributable to the FLT, which has a $90^{\circ}$ phase difference in relation to STT. However, the trajectory is always an ellipse, independent of the FLT contribution.

bias voltage dependence, as shown in Fig. 1c, we might expect an important contribution from $\left(G_{+,-}-G_{-,+}\right) V_{\mathrm{b}}$, which implies the nonlinear bias dependence of the STT as it is observed in our 
a

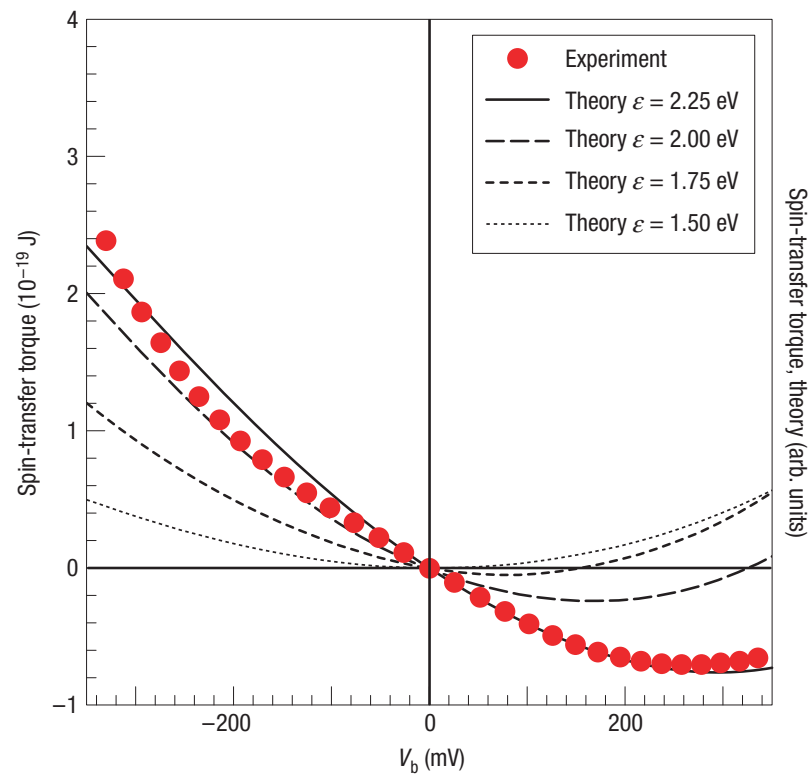

b

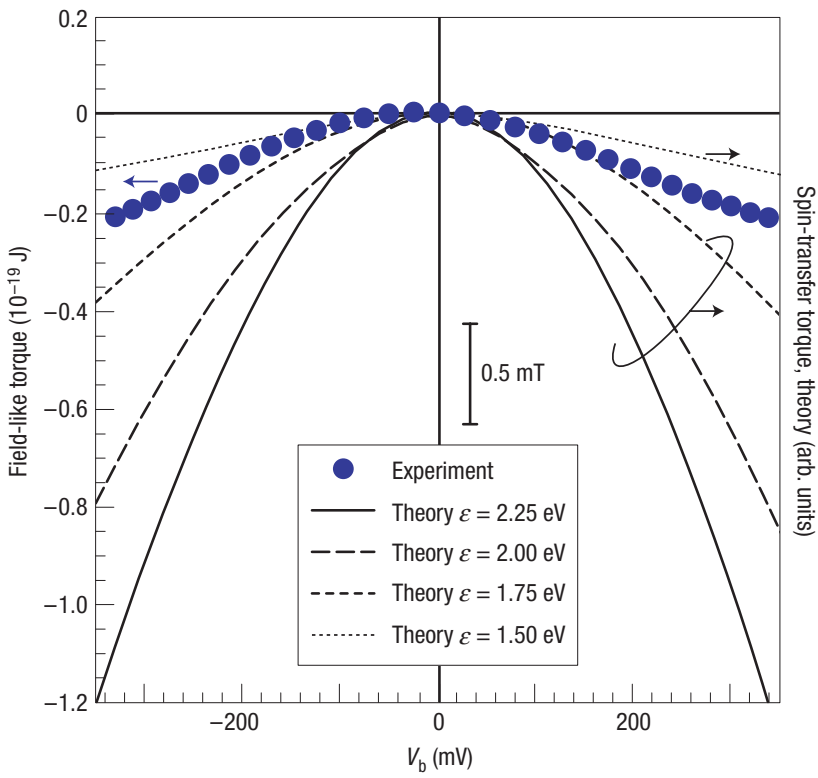

Figure 4 Bias dependence of the magnitudes of the spin torque. a, STT. b, FLT. The lines represent the theoretical results corresponding to different onsite exchange split parameters, $\varepsilon$ (ref. 10). The magnitude of the torque for experimental results is defined as spin torque $=(\hbar / 2) \beta_{\text {ST(F) }} l_{b} N \sin \theta_{12}=(\hbar / 2) N \sin \theta_{12} \int_{0}^{l_{b}} \beta_{\text {ST(F) }}^{\prime} \mathrm{d} l$, where $N$ is the total number of spins included in the FM-2 cell. Vertical axes (right-hand side axes) for the theoretical values show arbitrary units because of the different conductance of experiment and theory. Considering the conductance difference, the magnitudes of the observed spin torque show good agreement with theoretical values for both STT and FLT. In $\mathbf{b}$, the vertical line indicates the magnitude of the magnetic field corresponding to the FLT.

experiment. Extending that inference, Theodonis et al. calculated both STT and FLT (defined, respectively, as $T_{\|}$and $T_{\perp}$ in the literature) as a function of bias voltage taking exchange splitting, $\varepsilon$, as a parameter ${ }^{10}$. In Fig. 4, comparisons to theoretical predictions are also shown. Our STT data agree with those for $\varepsilon=2.25 \mathrm{eV}$, which is reasonable for a CoFe alloy. The data also predict a quadratic bias dependence of the FLT term, which agrees with our observations. Bending of the STT curve at $V_{\mathrm{b}}<-200 \mathrm{mV}$ in Fig. $4 \mathrm{a}$ is not reproduced by the theory. That feature might be related to dips observed in the $\mathrm{d} I / \mathrm{d} V$ spectra $^{23}$. Equation (3) suggests that an anomaly in conductance caused by the electronic band structure or magnon excited by injected spins ${ }^{24}$ imparts an influence on the spin torque, which is not considered in the theory. In addition, heating by the current might exert some effect. Reduction of $H_{\mathrm{d}}$ can enhance the last term in equation (2) when the sample temperature increases greatly.

Finally, we discuss the spin-torque-induced magnetization reversal (switching) mechanism of an M-RAM cell. Several groups have demonstrated switching in MgO-based $\mathrm{MTJs}^{7,16,25}$, thereby accelerating the development of M-RAMs with spin-torque writing $^{26,27}$. In our experiment, switching was observed at around $-270 \mathrm{mV}$ for the antiparallel-to-parallel transition, where the STT is large, as shown in Fig. 4a. On the other hand, switching from parallel to antiparallel was observed around $+380 \mathrm{mV}$, where the STT is small. Therefore, it is necessary to consider the contribution of the FLT for the parallel-to-antiparallel switching. Moreover, the angular dependence of the STT and FLT should be examined carefully. In addition to those remaining problems, theory suggests the possibility of controlling the spin-torque asymmetry by choosing material parameters. Therefore, we can increase the capability to control the switching properties of the memory cells by optimizing them.

Note added in proof. During the preparation and editing of this paper, torque measurement data using the same effect for a parallel alignment of magnetizations were reported by J. C. Sankey et al. Measurement of the spin-transfer-torque vector in magnetic tunnel junctions. Preprint at <http://jp.arxiv.org/abs/0705.4207> (2007).

\section{METHODS}

\section{SAMPLE PREPARATION}

The entire structure of the prepared film is $\mathrm{MgO}$ substrate/Ta/CuN/Ta/Ir$\mathrm{Mn} / \mathrm{Co}_{70} \mathrm{Fe}_{30} / \mathrm{Ru} / \mathrm{Co}_{60} \mathrm{Fe}_{20} \mathrm{~B}_{20} / \mathrm{MgO} / \mathrm{Co}_{60} \mathrm{Fe}_{20} \mathrm{~B}_{20} / \mathrm{Ta} / \mathrm{Ru}$. The $\mathrm{Co}-\mathrm{Fe} / \mathrm{Ru} / \mathrm{Co}-\mathrm{Fe}-\mathrm{B}$ is a synthetic ferrimagnet structure, in which magnetizations of $\mathrm{Co}-\mathrm{Fe}$ and $\mathrm{Co}-\mathrm{Fe}-\mathrm{B}$ align in an antiparallel configuration. The magnetization of $\mathrm{Co}-\mathrm{Fe}$ is pinned unidirectionally by an exchange-biasing field from the Pt-Mn antiferromagnetic layer. This hybrid structure is commonly used in magnetoresistive devices to harden the magnetization of the reference layer. The $\mathrm{MgO}$ substrate suppresses high-frequency losses due to currents through the substrate.

Tunnel junctions were fabricated using optical and electron beam lithography combined with an Ar-ion etching technique and a lift-off process. First, a bottom electrode is patterned using photolithography, with subsequent Ar-ion etching. Second, in the centre of the bottom electrode, an MTJ cell is prepared using electron beam lithography and Ar-ion etching. The rectangular cell is roughly $70 \mathrm{~nm} \times 250 \mathrm{~nm}$. Third, the entire sample surface is passivated by a thick $\mathrm{SiO}_{2}$ film. It is then partially removed using the lift-off technique to produce a contact hole. Finally, a $\mathrm{Cr} / \mathrm{Au}$ double layer top lead is fabricated using sputter deposition and Ar-ion etching. The widths of both the bottom and top leads are $4 \mu \mathrm{m}$.

\section{SPIN-TORQUE DIODE MEASUREMENT}

A low-frequency oscillator $(10 \mathrm{kHz})$ modulated the amplitude of the high-frequency current applied to the MTJ. The modulated component of the voltage signal across the MTJ was measured using a lock-in amplifier ( $\left.V_{\text {diode }}\right)$. High-frequency current power $(-15 \mathrm{dBm})$ was kept constant and the frequency was scanned from $50 \mathrm{MHz}$ to $15 \mathrm{GHz}$. During measurement, the d.c. bias current was applied to the sample, although the bias voltage $\left(V_{\mathrm{b}}\right)$ was measured using a digital voltmeter. A magnetic field $(H)$ of -400 Oe was applied in the in-plane direction with an angle $\left(\theta_{\mathrm{H}}\right)$ of $45^{\circ}$ with respect to the MTJ cell's long axis.

We measured $V_{\text {diode }}$ as a function of the frequency of the applied highfrequency current for different bias voltages. The $V_{\text {diode }}$ spectrum showed a resonance peak around $6.7 \mathrm{GHz}$, where a ferromagnetic resonance of the 
free-layer magnetization takes place. The peak shape is expressed as equation (1) in the main text as

$$
\begin{gathered}
f\left[\omega ; \beta_{\mathrm{ST}}, \beta_{\mathrm{FT}}, \omega_{0}, \Delta, I_{\mathrm{b}}, H, H_{\mathrm{d}}, \theta_{12}\right]=\operatorname{Re}\left[\frac{\omega_{a a} \beta_{\mathrm{FT}}^{\prime}-\left(i \omega+\omega_{\mathrm{ST}}\right) \beta_{\mathrm{ST}}^{\prime}}{\left(\omega_{0}^{2}-\omega^{2}\right)+i \omega \Delta}\right], \\
\beta_{\mathrm{FT}}^{\prime}=\left[\frac{\mathrm{d}}{\mathrm{d} I}\left(\beta_{\mathrm{FT}} I\right)\right]_{I=I_{\mathrm{b}}}, \\
\beta_{\mathrm{ST}}^{\prime}=\left[\frac{\mathrm{d}}{\mathrm{d} I}\left(\beta_{\mathrm{ST}} I\right)\right]_{I=I_{\mathrm{b}}} \\
\omega_{a a}=-\gamma\left(H \cos \left(\theta_{2}-\theta_{\mathrm{H}}\right)+H_{\mathrm{d}}\right), \\
\omega_{\mathrm{ST}}=\beta_{\mathrm{ST}} I_{\mathrm{b}} \cos ^{2} \theta_{12},
\end{gathered}
$$

where $\gamma$ is the gyromagnetic ratio $\left(-1.76 \times 10^{11} \mathrm{~T}^{-1} \mathrm{~s}^{-1}\right), \omega_{0}$ is the resonance frequency and $\Delta$ is the peak width, which is dominated by the damping factor, $\alpha$. Using the macrospin model, $\omega_{0}$ and $\Delta$ can be expressed as

$$
\begin{gathered}
\omega_{0}^{2}=\omega_{a a} \omega_{b b}+\omega_{\mathrm{ST}}^{2}, \\
\Delta=\alpha\left(\omega_{a a}+\omega_{b b}\right)+2 \omega_{\mathrm{ST}}, \\
\omega_{b b}=-\gamma H \cos \left(\theta_{12}-\theta_{\mathrm{H}}\right) .
\end{gathered}
$$

When $\left|\beta_{\mathrm{ST}}\right| \gg\left|\beta_{\mathrm{FT}}\right|$, a symmetric peak can be observed; in the opposite case, an antisymmetric peak is observed. The resistance values for $R_{\mathrm{P}}, R_{\mathrm{AP}}$ and $R_{0}$ include the electrical lead resistance.

Received 18 July 2007; accepted 15 October 2007; published 25 November 2007.

\section{References}

1. Slonczewski, J. C. Current-driven excitation of magnetic multilayers. J. Magn. Magn. Mater. 159, L1-L7 (1996).

2. Berger, L. Emission of spin waves by a magnetic multilayer traversed by a current. Phys. Rev. B 54 , 9353-9358 (1996).

3. Myers, E. B., Ralph, D. C., Katine, J. A., Louie, R. N. \& Buhrman, R. A. Current-induced switching of domains in magnetic multilayer devices. Science 285, 867-870 (1999).

4. Tsoi, M. et al. Excitation of a magnetic multilayer by an electric current. Phys. Rev. Lett. 80, 4281-4284 (1998).

5. Huai, Y., Albert, F., Nguyen, P., Pakala, M. \& Valet, T. Observation of spin-transfer switching in deep submicron-sized and low-resistance magnetic tunnel junctions. Appl. Phys. Lett. 84, 3118-3120 (2004)

6. Fuchs, G. D. et al. Spin-transfer effects in nanoscale magnetic tunnel junctions. Appl. Phys. Lett. 85 , 1205-1207 (2004).
7. Kubota, H. et al. Dependence of spin-transfer switching current on free layer thickness in $\mathrm{Co}-\mathrm{Fe}-\mathrm{B} / \mathrm{MgO} / \mathrm{Co}-\mathrm{Fe}-\mathrm{B}$ magnetic tunnel junctions. Appl. Phys. Lett. 89, 032505 (2006).

8. Kiselev, S. I. et al. Microwave oscillations of a nanomagnet driven by a spin-polarized current. Nature 425, 380-383 (2003).

9. Slonczewski, J. C. \& Sun, J. Z. Theory of voltage-driven current and torque in magnetic tunnel junctions. J. Magn. Magn. Mater. 310, 169-175 (2007).

10. Theodonis, I., Kioussis, N., Kalitsov, A., Chshiev, M. \& Butler, W. H. Anomalous bias dependence of spin torque in magnetic tunnel junctions. Phys. Rev. Lett. 97, 237205 (2006).

11. Yuasa, S., Nagahama, T., Fukushima, A., Suzuki, Y. \& Ando, K. Giant room-temperature magnetoresistance in single-crystal Fe/MgO/Fe magnetic tunnel junctions. Nature Mater. 3, 868-871 (2004).

12. Parkin, S. S. P. et al. Giant tunnelling magnetoresistance at room temperature with $\mathrm{MgO}$ (100) tunnel barriers. Nature Mater. 3, 862-867 (2004).

13. Djayaprawira, D. D. et al. $230 \%$ room-temperature magnetoresistance in $\mathrm{CoFeB} / \mathrm{MgO} / \mathrm{CoFeB}$ magnetic tunnel junctions. Appl. Phys. Lett. 86, 092602 (2005).

14. Butler, W. H., Zhang, X.-G., Schulthess, T. C. \& MacLaren, J. M. Spin-dependent tunneling conductance of Fe $|\mathrm{MgO}| \mathrm{Fe}$ sandwiches. Phys. Rev. B 63, 054416 (2001).

15. Zhang, S., Levy, P. M., Marley, A. C. \& Parkin, S. S.P. Quenching of magnetoresistance by hot electrons in magnetic tunnel junctions. Phys. Rev. Lett. 79, 3744-3747 (1997).

16. Hayakawa, J. et al. Current-driven magnetization switching in $\mathrm{CoFeB} / \mathrm{MgO} / \mathrm{CoFeB}$ magnetic tunnel junctions. Jpn. J. Appl. Phys. 2 44, L1267-L1270 (2005).

17. Slonczewski, J. C. Conductance and exchange coupling of two ferromagnets separated by a tunneling barrier. Phys. Rev. B 39, 6995-7002 (1989).

18. Tulapurkar, A. A. et al. Spin-torque diode effect in magnetic tunnel junctions. Nature 438 339-342 (2005).

19. Sankey, J. C. et al. Spin-transfer-driven ferromagnetic resonance of individual nanomagnets. Phys. Rev. Lett. 96, 277601 (2006).

20. Zhang, S., Levy, P. M. \& Fert, A. Mechanisms of spin-polarized current-driven magnetization switching. Phys. Rev. Lett. 88, 263301 (2002).

21. Suzuki, Y. et al. Microwave properties of spin injections devices. Magnet. Japan 2, 282-290 (2007).

22. Slonczewski, J. C. Currents, torques, and polarization factors in magnetic tunnel junctions. Phys. Rev. B 71, 024411 (2005).

23. Matsumoto, R. et al. Tunneling spectra of sputter-deposited $\mathrm{CoFeB} / \mathrm{MgO} / \mathrm{CoFeB}$ magnetic tunnel junctions showing giant tunneling magnetoresistance effect. Solid State Commun. 136, 611-615 (2005).

24. Levy, P. M. \& Fert, A. Spin transfer in magnetic tunnel junctions with hot electrons. Phys. Rev. Lett. 97, 097205 (2006).

25. Diao, Z. et al. Spin transfer switching and spin polarization in magnetic tunnel junctions with $\mathrm{MgO}$ and $\mathrm{AlO}_{x}$ barriers. Appl. Phys. Lett. 87, 232502 (2005).

26. Hosomi, M. et al. International Electron Devices Meeting 459-462 (IEEE, Washington, 2005).

27. Kawahara, T. et al. International Solid-State Circuits Conference 480-481 (IEEE, San Francisco, 2007).

\section{Acknowledgements}

This work was partially supported by the New Energy Development Organization (NEDO) and the 21 st COE program by JSPS. We thank D. Ralph for a useful comment on the derivation of the spin torque.

Correspondence and requests for materials should be addressed to H.K.

Supplementary Information accompanies this paper on www.nature.com/naturephysics.

\section{Author contributions}

Y.N., K.T., D.D. and N.W. optimized the sputtering process for MgO-based magnetic tunnel junctions with low resistance and high magnetoresistance. A.F. and K.Y. designed the sample geometry and carried out microfabrication. H.K. and H.M. carried out the measurements and the analysis with help from T.N.; Y.S. developed the theoretical model for the analysis. H.K. prepared the manuscript with review and input from Y.S., S.Y. and K.A.

Reprints and permission information is available online at http://npg.nature.com/reprintsandpermissions/ 